\title{
Coherence-induced entanglement
}

\author{
Fu-li Li, Han Xiong, and M. Suhail Zubairy \\ Institute for Quantum Studies and Department of Physics, Texas A\&M University, College Station, Texas 77843-4242, USA
}

(Received 7 March 2005; published 19 July 2005)

\begin{abstract}
We show how atomic coherence can lead to entanglement between two thermal fields at a temperature $T$. We first show that the passage of a three-level atom in $V$ configuration without coherence cannot create entanglement. However, if the excited states are driven by a microwave field, the resulting atomic coherence can lead to entanglement between the thermal fields. We show that, no matter how high the temperature of the fields is, the thermal fields can always be entangled in the presence of atomic coherence.
\end{abstract}

DOI: 10.1103/PhysRevA.72.010303

PACS number(s): 03.67.Mn, 03.65.Ud

Atomic coherence [1], which results from a coherent superposition of different states of a single atom, can lead to many different quantum optical phenomena. These include correlated spontaneous emission laser [2], lasing without inversion [3], electromagnetically induced transparence [4] and spontaneous emission cancellation [5]. Atomic coherence has recently been shown to play a key role in quantum thermodynamics. For example, Scully et al. showed that mechanical work may be extracted from a single heat bath via vanishing atomic coherence [6].

Another feature of quantum systems is entanglement that refers to quantum correlation among subsystems that share a common quantum state. Quantum entanglement plays an essential role in quantum information processing such as quantum computation [7], quantum teleportation [8], and quantum cryptography [9].

In this paper we discuss an important application where atomic coherence plays a crucial role in creating entanglement between two modes of the electromagnetic field inside a doubly resonant cavity at temperature $T$, which are coupled to two transitions of an atom in $V$ configuration. The two important concepts, entanglement and atomic coherence, are shown to be closely related.

In earlier studies on the interaction of thermal fields with the atomic systems, it has been shown that atom-field [10] and atom-atom [11] entanglement can be generated in such systems. In these studies, at least, one subsystem is initially in a pure state. The entanglement appears only when the atom and the field are not in thermal equilibrium. Here we show that atomic coherence is the unique resource of creating entanglement between two cavity modes in thermal state even at arbitrarily high temperature.

The model under consideration is shown in Fig. 1. We consider the interaction of an atom in the $V$ configuration with the fields inside a cavity at temperature $T$. Here we assume that the transitions between the upper levels $|a\rangle$ and $|b\rangle$ to the ground state $|c\rangle$ are dipole allowed and these transitions are coupled resonantly with the modes inside the cavity. The transition between the upper levels $|a\rangle$ and $|b\rangle$ is dipole forbidden, while the coherence between level $|a\rangle$ and level $|b\rangle$ could be created by applying a classical magnetic field between these two levels. The interaction picture Hamiltonian of the system is given by

$$
\hat{H}=\hbar g_{1}\left(|a\rangle\left\langle c\left|\hat{a}_{1}+\right| c\right\rangle\langle a| \hat{a}_{1}^{\dagger}\right)+\hbar g_{2}\left(|b\rangle\left\langle c\left|\hat{a}_{2}+\right| c\right\rangle\langle b| \hat{a}_{2}^{\dagger}\right),
$$

where $\hat{a}_{1}\left(\hat{a}_{1}^{\dagger}\right)$ and $\hat{a}_{2}\left(\hat{a}_{2}^{\dagger}\right)$ are the annihilation (creation) operators for the two cavity modes and $g_{1,2}$ are coupling constants of the atom with the fields.

We consider the initial states of the cavity fields to be diagonal in the Fock-state representation and the atom to be prepared in a coherent superposition of the upper levels by a classical field of frequency $\omega_{a b}$ as shown in Fig. 1. The initial state of the atom-field system is written as

$$
\begin{aligned}
\hat{\rho}_{a f}(0)= & \sum_{n_{1}=0}^{\infty} P_{n_{1}}\left|n_{1}\right\rangle\left\langle n_{1}\left|\otimes \sum_{n_{2}=0}^{\infty} P_{n_{2}}\right| n_{2}\right\rangle\left\langle n_{2}\right| \otimes\left(\rho_{a a}|a\rangle\langle a|\right. \\
& \left.+\rho_{b b}|b\rangle\left\langle b\left|+\rho_{c c}\right| c\right\rangle\langle c|+\rho_{a b}\langle a|\left\langle b\left|+\rho_{b a}\right| b\right\rangle\langle a|\right),
\end{aligned}
$$

where $P_{n_{1,2}}$ are the probabilities for having photon number states $\left|n_{1,2}\right\rangle$. An example of fields with vanishing offdiagonal matrix elements in the Fock-state representation is a

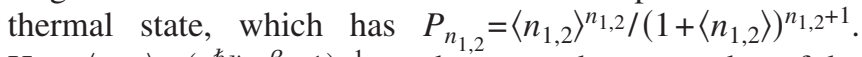
Here $\left\langle n_{1,2}\right\rangle=\left(e^{\hbar \nu_{1,2} \beta}-1\right)^{-1}$ are the mean photon number of the fields at temperature $T$ with $\nu_{1,2}$ being the field frequencies, and $\beta^{-1}=k_{B} T$ with $k_{B}$ being the Boltzmann constant.

The density matrix operator at time $\mathrm{t}$ is given by $\hat{\rho}_{a f}(t)$ $=\hat{U}(t) \hat{\rho}_{a f}(0) \hat{U}^{\dagger}(t)$ where $\hat{U}(t)=\exp (-i \hat{H} t / \hbar)$ is the time evolution operator. It follows, on taking a trace over the atomic variables, that the reduced density matrix operator for the fields is given by

$$
\begin{aligned}
\hat{\rho}_{f}(t)= & \sum_{n_{1}=0}^{\infty} \sum_{n_{2}=0}^{\infty} \rho_{n_{1}, n_{2} ; n_{1}, n_{2}}\left|n_{1}, n_{2}\right\rangle\left\langle n_{1}, n_{2}\right| \\
& +\rho_{a b} \sum_{n_{1}=0}^{\infty} \sum_{n_{2}=0}^{\infty} \rho_{n_{1}+1, n_{2} ; n_{1}, n_{2}+1}\left|n_{1}+1, n_{2}\right\rangle\left\langle n_{1}, n_{2}+1\right| \\
& +\rho_{b a} \sum_{n_{1}=0}^{\infty} \sum_{n_{2}=0}^{\infty} \rho_{n_{1}, n_{2}+1 ; n_{1}+1, n_{2}}\left|n_{1}, n_{2}+1\right\rangle\left\langle n_{1}+1, n_{2}\right|,
\end{aligned}
$$

where the matrix elements are given by 


$$
\begin{aligned}
\rho_{n_{1}, n_{2} ; n_{1}, n_{2}}= & P_{n_{1}} P_{n_{2}}\left\{\rho_{a a}\left[1-g_{1}^{2}\left(n_{1}+1\right) A_{n_{1}+1, n_{2}}\left(1-C_{n_{1}+1, n_{2}}\right)\right]^{2}+\rho_{b b}\left[1-g_{2}^{2}\left(n_{2}+1\right) A_{n_{1}, n_{2}+1}\left(1-C_{n_{1}, n_{2}+1}\right)\right]^{2}\right\} \\
& +g_{1}^{2} g_{2}^{2}\left\{\rho_{a a} P_{n_{1}-1} P_{n_{2}+1} n_{1}\left(n_{2}+1\right) \times A_{n_{1}, n_{2}+1}^{2}\left(1-C_{n_{1}, n_{2}+1}\right)^{2}+\rho_{b b} P_{n_{1}+1} P_{n_{2}-1} n_{2}\left(n_{1}+1\right) A_{n_{1}+1, n_{2}}^{2}\left(1-C_{n_{1}+1, n_{2}}\right)^{2}\right\} \\
& +\left\{\rho_{a a} P_{n_{1}-1} P_{n_{2}} g_{1}^{2} n_{1}+\rho_{b b} P_{n_{1}} P_{n_{2}-1} g_{2}^{2} n_{2}\right\} A_{n_{1}, n_{2}} S_{n_{1}, n_{2}}^{2}+\rho_{c c}\left\{P_{n_{1}} P_{n_{2}} C_{n_{1}, n_{2}}^{2}+P_{n_{1}+1} P_{n_{2}} g_{1}^{2}\left(n_{1}+1\right) A_{n_{1}+1, n_{2}} S_{n_{1}+1, n_{2}}^{2}\right. \\
& \left.+P_{n_{1}} P_{n_{2}+1} g_{2}^{2}\left(n_{2}+1\right) A_{n_{1}, n_{2}+2} S_{n_{1}, n_{2}+1}^{2}\right\} \\
& \\
\rho_{n_{1}+1, n_{2} ; n_{1}, n_{2}+1} & -g_{1} g_{2} \sqrt{\left(n_{1}+1\right)\left(n_{2}+1\right)} \times\left\{A_{n_{1}+1, n_{2}+1}\left(1-C_{n_{1}+1, n_{2}+1}\right) \times\left(P_{n_{1}+1} P_{n_{2}}\left[1-g_{1}^{2}\left(n_{1}+2\right) A_{n_{1}+2, n_{2}}\left(1-C_{n_{1}+2, n_{2}}\right)\right]\right.\right. \\
& \left.\left.+P_{n_{1}} P_{n_{2}+1}\left[1-g_{2}^{2}\left(n_{2}+2\right) A_{n_{1}, n_{2}+2}\left(1-C_{n_{1}, n_{2}+2}\right)\right]\right)-P_{n_{1}} P_{n_{2}} \sqrt{A_{n_{1}+1, n_{2}} A_{n_{1}, n_{2}+1}} S_{n_{1}+1, n_{2}} S_{n_{1}, n_{2}+1}\right\},
\end{aligned}
$$

$$
\rho_{n_{1}, n_{2}+1 ; n_{1}+1, n_{2}}=\left(\rho_{n_{1}+1, n_{2} ; n_{1}, n_{2}+1}\right)^{*},
$$

with $A_{n_{1}, n_{2}}=\left(g_{1}^{2} n_{1}+g_{2}^{2} n_{2}\right)^{-1}, \quad S_{n_{1}, n_{2}}=\sin \left(\sqrt{g_{1}^{2} n_{1}+g_{2}^{2} n_{2}} t\right)$ and $C_{n_{1}, n_{2}}=\cos \left(\sqrt{g_{1}^{2} n_{1}+g_{2}^{2} n_{2}} t\right)$.

We first discuss the case where initially there is no atomic coherence, i.e., $\rho_{a b}=\rho_{b a}=0$. It is clear from Eq. (3) that, under this condition, the fields are definitely in a separable state,

$$
\hat{\rho}_{f}(t)=\sum_{n_{1}=0}^{\infty} \sum_{n_{2}=0}^{\infty} \rho_{n_{1}, n_{2} ; n_{1}, n_{2}}\left|n_{1}, n_{2}\right\rangle\left\langle n_{1}, n_{2}\right| .
$$

In this case, the fields may still have classical statistical correlation if $\rho_{n_{1}, n_{2} ; n_{1}, n_{2}}$ cannot be decomposed into a direct product of the form $\rho_{n_{1}, n_{1}} \otimes \rho_{n_{2}, n_{2}}$. As a special case, we consider the situation where the atom and the fields are initially in thermal equilibrium. In this case, the level populations of the atom are determined by the relations $\rho_{a a} / \rho_{c c}$ $=\left\langle n_{1}\right\rangle /\left(\left\langle n_{1}\right\rangle+1\right)$ and $\rho_{b b} / \rho_{c c}=\left\langle n_{2}\right\rangle /\left(\left\langle n_{2}\right\rangle+1\right)$. It follows, on substituting these relations in Eq. (4), that state (7) becomes

$$
\rho_{f}=\sum_{n_{1}=0}^{\infty} P_{n_{1}}\left|n_{1}\right\rangle\left\langle n_{1}\left|\otimes \sum_{n_{2}=0}^{\infty} P_{n_{2}}\right| n_{2}\right\rangle\left\langle n_{2}\right|,
$$

i.e., we have neither entanglement nor classical correlation between the fields as the two fields are completely separable.

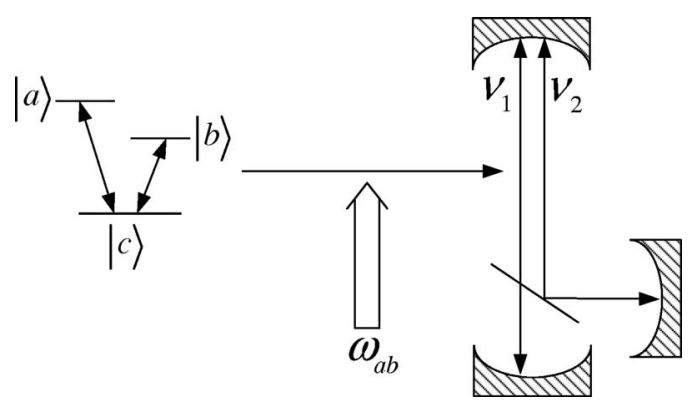

FIG. 1. A three-level atom in $V$ configuration with initial populations $\rho_{a a}, \rho_{b b}, \rho_{c c}$ is prepared in a superposition of upper levels $|a\rangle$ and $|b\rangle$ by a resonant classical field. The atom then passes through a doubly resonant cavity that is resonant with $|a\rangle-|c\rangle$ and $|b\rangle-|c\rangle$ transitions. The fields inside the cavity are initially diagonal, such as a thermal state.
So how do we entangle the thermal fields? We show that this can be accomplished via atomic coherence.

In Eq. (4), the term proportional to the population of the level $|c\rangle$ results from one-photon absorption processes. The photon absorption processes lead to the classical correlation between the fields and have no contribution to the entanglement. Therefore, in order to create strong entanglement, the population of the level $|c\rangle$ should be reduced. In Eq. (5), the terms related to $P_{n_{1}+1} P_{n_{2}}$ and $P_{n_{1}} P_{n_{2}+1}$ involve the processes in which one mode photon is emitted and another mode photon is absorbed, and the term proportional to $P_{n_{1}} P_{n_{2}}$ comes from the photon emission processes of the upper levels. If the atomic coherence exists, these terms contribute to the off-diagonal matrix elements. Without the off-diagonal correlation contribution given in Eqs. (5) and (6), the fields have no entanglement. However, the existence of the off-diagonal correlation can not guarantee entanglement. Thus, we need to find a condition for the existence of entanglement between the fields in the state (3).

State (3) is defined in an infinite dimensional Hilbert space. In general, it is very difficult to measure the entanglement in such systems $[10,11]$. However we recall that entanglement cannot be generated through local transformations. To estimate the entanglement of (3), we consider the local projection operators $\hat{A}_{n_{1}}=\left|n_{1}\right\rangle\left\langle n_{1}|+| n_{1}+1\right\rangle\left\langle n_{1}+1\right|$ and $\hat{B}_{n_{2}}=\left|n_{2}\right\rangle\left\langle n_{2}|+| n_{2}+1\right\rangle\left\langle n_{2}+1\right|$ with $n_{1,2}=0,2,4, \ldots$ If the fields are in a separable state $\Sigma_{i} p_{i} \rho_{i}^{(1)} \otimes \rho_{i}^{(2)}$, the projected state $\hat{A}_{n_{1}} \hat{B}_{n_{2}} \sum_{i} p_{i} \rho_{i}^{(1)} \otimes \rho_{i}^{(2)} \hat{B}_{n_{2}}^{\dagger} \hat{A}_{n_{1}}^{\dagger}$ is still separable. Then we can claim the existence of entanglement in (3) if the entanglement exists in the projected state.

The projection of (3) on the subspace spanned by basis vectors $\left(\left|n_{1}\right\rangle,\left|n_{1}+1\right\rangle\right) \otimes\left(\left|n_{2}\right\rangle,\left|n_{2}+1\right\rangle\right)$ with fixed photon numbers $n_{1,2}(=0,2,4, \ldots)$ leads to the state

$$
\left[\hat{\rho}_{f}(t)\right]_{n_{1}, n_{2}}=\hat{A}_{n_{1}} \hat{B}_{n_{2}} \hat{\rho}_{f}(t) \hat{B}_{n_{2}}^{\dagger} \hat{A}_{n_{1}}^{\dagger} .
$$

In the subspace under consideration, the projected density matrix operator (9) becomes a $4 \times 4$ Hermitian matrix. Now we can apply the Peres-Horodecki sufficiency condition [12] for the inseparability of density matrices of a two-party quantum system. For a bipartite system, a state is separable if and only if its density matrix can be written as $\rho_{A B}=\Sigma_{i} p_{i} \rho_{A}^{i}$ $\otimes \rho_{B}^{i}$, where $p_{i} \geqslant 0$ and $\Sigma_{i} p_{i}=1$. It is clear that the partial 


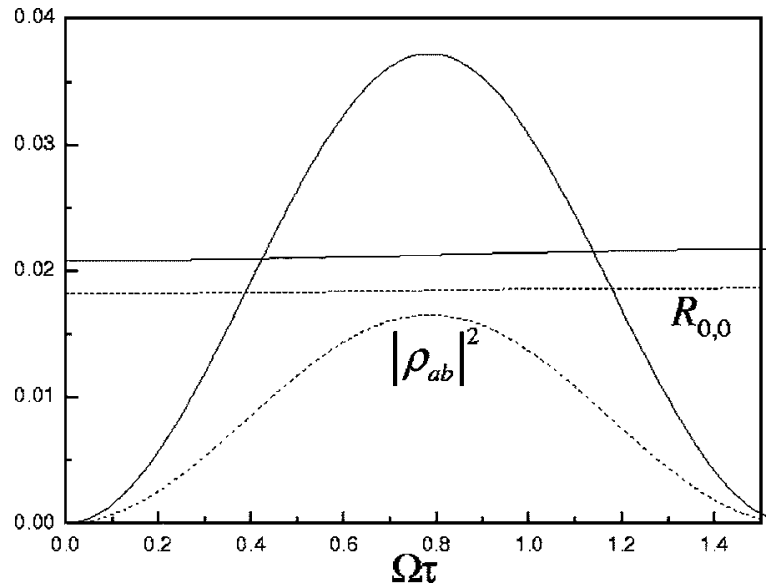

FIG. 2. The solid lines are for the case with $\left\langle n_{1}\right\rangle=0.1$ and $\left\langle n_{2}\right\rangle$ =5.0. The dashed lines are for the case with $\left\langle n_{1}\right\rangle=0.1$ and $\left\langle n_{2}\right\rangle$ $=1.0 . g t=11.0$.

transposed matrix $\sigma_{A B}=\Sigma_{i} p_{i} \rho_{A}^{i} \otimes\left(\rho_{B}^{i}\right)^{T}$ of a separable state has only non-negative eigenvalues. According to the PeresHorodecki criterion, if the partial transposed matrix $\sigma_{A B}$ of a state has negative eigenvalues, this state must be entangled.

The partial transposition of the density matrix (9) has a negative eigenvalue if the condition

$$
\left|\rho_{a b}\right|^{2}>R_{n_{1}, n_{2}}=\frac{\rho_{n_{1}, n_{2} ; n_{1}, n_{2}} \rho_{n_{1}+1, n_{2}+1 ; n_{1}+1, n_{2}+1}}{\left|\rho_{n_{1}+1, n_{2} ; n_{1}, n_{2}+1}\right|^{2}}
$$

is satisfied. According to the Peres-Horodecki condition, we can claim that state (3) is an entangled state if the condition (10) is satisfied. This kind of methodology to detect the entanglement of an infinite dimensional mixed state for a bipartite system was discussed and used by Bose et al. in [10].

An experimental verification of inequality (10) requires the full knowledge of the state. Several schemes have been proposed to reconstruct a two-mode state in a high- $Q$ cavity recently [13]. For example, one can look at the spontaneous emission spectrum in a driven four-level atomic system passing through the cavity to recover the Wigner function of the

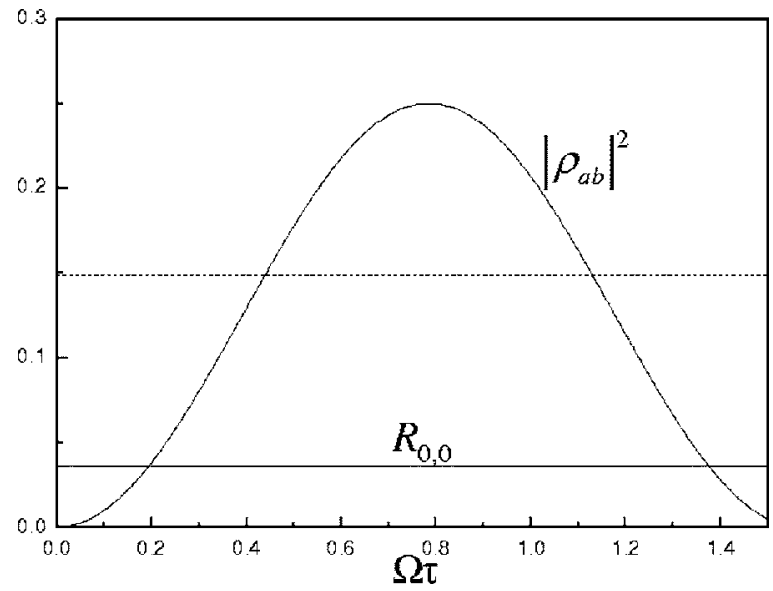

FIG. 3. The solid lines are for the case with $\left\langle n_{1}\right\rangle=\left\langle n_{2}\right\rangle=1.0$. The dashed line is for the case with $T \rightarrow \infty$. $g t=5.0$, and $\rho_{a a}=1$.

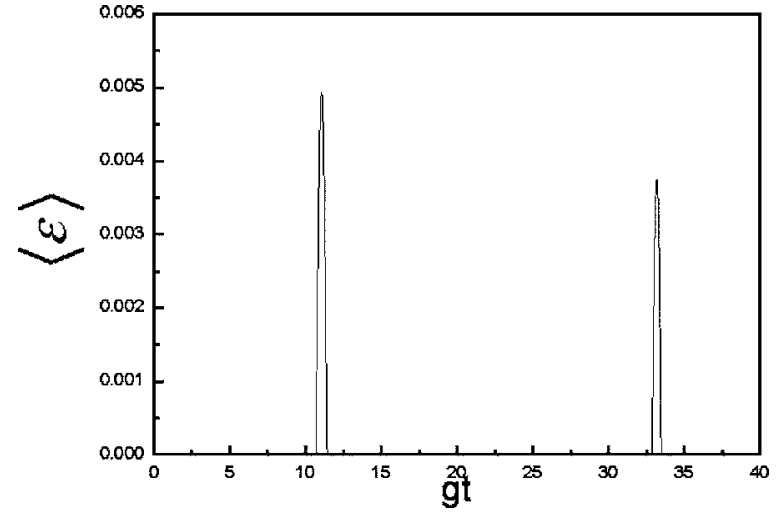

FIG. 4. The time evolution of the entanglement measurement with $\left\langle n_{1}\right\rangle=0.1,\left\langle n_{2}\right\rangle=5.0$, and $\Omega \tau=\pi / 4$.

two-mode field [14]. Once the Wigner function is known, the right-hand side of (10) can be calculated in a straightforward manner.

It follows from Eqs. (4) and (5) that $R_{0,0}=0$ when $\left\langle n_{1}\right\rangle$ and $\left\langle n_{2}\right\rangle$ approach zero. Thus, for this case, arbitrarily small but nonzero atomic coherence can induce entanglement between the two modes. For a general case, $R_{n_{1}, n_{2}}$ is always larger than zero. Therefore there is the minimum atomic coherence beyond which the entanglement can appear. The right side of (10) depends on the level populations that satisfy the physical restriction with respect to the atomic coherence: $\rho_{a a} \rho_{b b}$ $\geqslant\left|\rho_{a b}\right|^{2}$. In order to conveniently control the population and atomic coherence at the same time, we consider the atom whose level populations initially are $\rho_{i i}(0)(i=a, b, c)$ and off-diagonal matrix elements $\rho_{i j}=0$ for $i \neq j$. A coherence between the excited states $a$ and $b$ is created when the atom interacts resonantly with a classical magnetic field (since this transition is dipole forbidden) of frequency $\omega_{a b}$ for a time $\tau$. After the interaction with the classical field, the poulations and the atomic coherence are given by [15]

$$
\begin{gathered}
\rho_{a a}=\rho_{a a}(0) \cos ^{2}(\Omega \tau)+\rho_{b b}(0) \sin ^{2}(\Omega \tau), \\
\rho_{b b}=\rho_{a a}(0) \sin ^{2}(\Omega \tau)+\rho_{b b}(0) \cos ^{2}(\Omega \tau), \\
\rho_{a b}=\left(\rho_{b a}\right)^{*}=i e^{i \theta}\left[\rho_{a a}(0)-\rho_{b b}(0)\right] \sin (\Omega \tau) \cos (\Omega \tau),
\end{gathered}
$$

where $\Omega$ is the Rabi frequency and $\theta$ is the phase of the driving field. All the other density matrix elements remain unchanged. In this way, we can unitarily and continuously control the level populations and atomic coherence by use of the single parameter $\Omega \tau$. After passing through the classical field, the atom acquires a coherence. When this atom passes through the cavity with two thermal fields, the state of the fields is described by the density matrix (3). The entanglement of the resulting state of the field is determined by the condition (10).

In Fig. 2, the right side of (10) with $n_{1}=n_{2}=0$ and the squared modulus of the atomic coherence (11) are shown as a function of $\Omega \tau$ when the atom and the fields are initially in thermal equilibrium. In the present calculation, we take $g_{1}$ 
$=g_{2}=g$. We also find that, as a function of $n_{1}$ and $n_{2}$, the right side of the inequality (10) takes the minimal value with $n_{1}$ $=n_{2}=0$. From Fig. 2, it can be noticed that the entanglement condition (10) can be satisfied if the difference between the mean thermal photon numbers of the two fields is sufficiently large. This situation may not easily realizable because it requires a large frequency difference between the two upper levels.

Equation (11) shows that the atomic coherence is proportional to the population inversion of the upper levels. On the other hand, the numerator of the right side of the condition (10) decreases if the level populations $\rho_{a a}, \rho_{b b}$, or $\rho_{c c}$ are small. Therefore, the best initial condition of the atom for satisfying the condition (10) is that the atom is in one of the upper levels. For this case, Fig. 3 shows that the entanglement condition (10) can be satisfied even if the temperature becomes arbitrarily high.

As discussed earlier, the Hilbert space for the complete system is infinite dimensional, i.e., the dimension of the density matrix (3) is infinite. We can therefore obtain an infinite number of the projected $4 \times 4$ Hermitian matrices (9) with different photon numbers $n_{1}$ and $n_{2}$ by projecting the density matrix (3) into the subspaces. We can then use the quantity [16]

$$
\langle\mathcal{E}\rangle=-2 \sum_{n_{1}, n_{2}=0,2,4, \ldots}^{\infty} p_{n_{1}, n_{2}} \lambda_{n_{1}, n_{2}}
$$

to measure the entanglement of (3), where $\lambda_{n_{1}, n_{2}}$ is the negative eigenvalue of the partial transposed density matrix of (9) and $p_{n_{1}, n_{2}}=\rho_{n_{1}, n_{2} ; n_{1}, n_{2}}+\rho_{n_{1}+1, n_{2} ; n_{1}, n_{2}}$ $+\rho_{n_{1}, n_{2}+1 ; n_{1}, n_{2}}+\rho_{n_{1}+1, n_{2}+1 ; n_{1}+1, n_{2}+1}$ is the probability of taking the $4 \times 4$ matrix (9) out of the matrix (3). If $\langle\mathcal{E}\rangle=0$, it does not mean nonentanglement. If $\langle\mathcal{E}\rangle \neq 0$, however, we can ensure that the infinite dimensional density matrix (3) must be an entangled state. In Fig. 4, the time evolution of the entanglement (12) is shown when the atom and the fields are initially in thermal equilibrium. It is seen that for this case

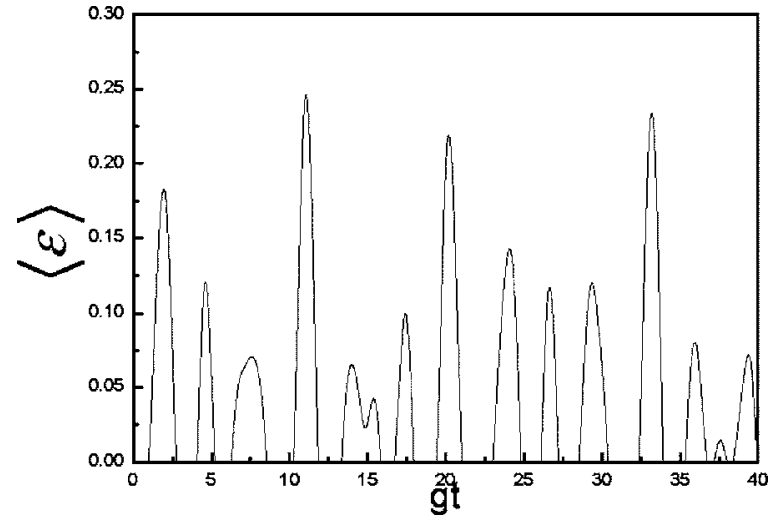

FIG. 5. The time evolution of the entanglement measurement with $\left\langle n_{1}\right\rangle=1.0,\left\langle n_{2}\right\rangle=1.0$, and $\Omega \tau=\pi / 4$ and $\rho_{a a}=1$.

the weak entanglement is detected at several time points. As pointed out earlier, the atomic coherence will become stronger when the atom is initially in one of the upper level. Therefore, we may expect that in this case the stronger entanglement will be detected. Figure 5 shows the time evolution of the entanglement (12) when the atom is initially in the level $\langle a\rangle$.

In conclusion we have shown that, no matter how high the temperature is, and the atom and the fields are initially in either thermal nonequilibrium or equilibrium, two thermal field modes in a cavity can be entangled by a single threelevel atom of the $V$ configuration when the coherence between two upper levels is beyond a critical value. The present result reveals a relation between entanglement and atomic coherence.

This research is supported by the Air Force Office of Scientific Research, DARPA-QuIST, Office of Naval Research, and the TAMU Telecommunication and Informatics Task Force (TITF) initiative.
[1] M. O. Scully and M. S. Zubairy, Quantum Optics (Cambridge, London, 1997).

[2] M. O. Scully, Phys. Rev. Lett. 55, 2802 (1985); M. O. Scully and M. S. Zubairy, Phys. Rev. A 35, 752 (1987).

[3] M. O. Scully et al., Phys. Rev. Lett. 62, 2813 (1989); O. Kocharovskaya, Phys. Rep. 219, 175 (1992); E. Arimondo, Prog. Opt. 35, 257 (1996); S. E. Harris, Phys. Rev. Lett. 62, 1033 (1989).

[4] S. E. Harris, Phys. Today 50, 36 (1997); S. E. Harris et al., Phys. Rev. Lett. 64, 1107 (1990); K.-J. Boller et al., ibid. 66, 2593 (1991).

[5] S. Y. Zhu and M. O. Scully, Phys. Rev. Lett. 76, 388 (1996); H. R. Xia et al., ibid. 77, 1032 (1996); H. Huang et al., Phys. Rev. A 55, 744 (1997).

[6] M. O. Scully et al., Science 299, 862 (2003).

[7] A. Ekert and R. Jozsa, Rev. Mod. Phys. 68, 733 (1996); E. Knill et al., Nature (London) 409, 46 (2001).
[8] C. H. Bennett et al., Phys. Rev. Lett. 70, 1895 (1993).

[9] A. K. Ekert, Phys. Rev. Lett. 67, 661 (1991).

[10] S. Bose et al., Phys. Rev. Lett. 87, 050401 (2000).

[11] M. S. Kim et al., Phys. Rev. A 65, 040101(R) (2002).

[12] A. Peres, Phys. Rev. Lett. 77, 1413 (1996); M. Horodecki et al., Phys. Lett. A 223, 1 (1996).

[13] H. Kühn et al., Phys. Rev. A 51, 4240 (1995); M. G. Raymer et al., ibid. 54, 2397 (1996); M. S. Kim and G. S. Agarwal, ibid. 59, 3044 (1999); G. M. D'Ariano et al., ibid. 61, 013806 (1999); J. Fiurasek, ibid. 63, 033806 (2001); M. Ahmad, S. Qamar, and M. S. Zubairy, ibid. 67, 043815 (2003).

[14] M. Ikram and M. S. Zubairy, Phys. Rev. A 65, 044305 (2002).

[15] M. S. Zubairy, in Quantum Limits to the Second Law: First International Conference, edited by D. P. Sheehan, AIP Conf. Proc. No. 643 (AIP, Melville, NY, 2002), pp. 92-95.

[16] J. Lee and M. S. Kim, Phys. Rev. Lett. 84, 4236 (2000). 